\title{
DIFERENCIAS SEXUALES EN LA PREVALENCIA E INTENSIDAD DE LOS MIEDOS DURANTE LA INFANCIA Y LA ADOLESCENCIA: DATOS BASADOS EN EL FSSC-R
}

\author{
ROSA M. VALIENTE, BONIFACIO SANDIN, PALOMA CHOROT y AURORA TABAR
}

Universidad Nacional de Educación a Distancia (UNED), Madrid

\begin{abstract}
El objetivo del presente trabajo ha consistido en estudiar las diferencias sexuales en prevalencia e intensidad de los miedos normativos en una larga muestra no clínica $(N=1080)$ de niños y adolescentes (con un rango de edad entre los 8 y los 18 años). Los participantes completaron la versión española del Fear Survey Schedule for Children-Revised (FSSC-R; Ollendick, 1983), una prueba designada para evaluar los miedos comunes de la infancia y la adolescencia. Los resultados obtenidos indicaron que: (1) las chicas informaron mayores niveles de prevalencia e intensidad de los miedos que los chicos; (2) tanto las chicas como los chicos refirieron elevados niveles en el número de miedos relativos a la dimensión de peligro físico y muerte; y (3) chicas y chicos coincidieron en 9 de los 10 miedos más comunes. En términos generales, estos resultados son muy similares a los datos obtenidos con otras muestras de cultura occidental (Estados Unidos, Australia e Inglaterra), y proporcionan evidencia en apoyo de la validez transcultural de la versión española del FSSC-R.
\end{abstract}

Palabras clave: Miedos, niños, adolescentes, diferencias sexuales.

Gender differences in prevalence and intensity of fears in a sample of children and adolescents: Data based on the FSSC-R

The aim of this work was to examine gender differences in prevalence and intensity of common fears in a large sample $(N=1080)$ of nonclinical children and adolescents (ranging in age from 8 to 18 years). Participants completed the Spanish version of the Fear Survey Schedule for Children-Revised (FSSC-R; Ollendick, 1983), a validated measure designed to assess common fears in children an adolescents. Results showed that: (1) girls reported significantly higher levels of fearfulness (prevalence and intensity of fears) than boys; (2) both girls and boys reported high levels of number of fears on the physical danger and death domain (nine of the ten most common fears belong to this fear dimension); and (3) both girls and boys endorsed 9 of the 10 same fears as their highest fears. Overall, these data paralleled those reported in other Western samples (United States, Australia and England) and provide cross-cultural validity of the Spanish version of the FSSC-R.

Key words: Fears, children, adolescents, gender differences.

\section{INTRODUCCIÓN}

Las fobias son fenómenos bastante frecuentes en la población general, tanto en personas adultas como en los niños y en los adolescentes (Sandin, 1997, 1999). Los miedos, si bien suelen tener una finalidad adaptativa durante ciertos periodos del desarrollo evolutivo, también pue-

Correspondencia: Rosa M. Valiente, Facultad de Pscilogía, Universidad Nacional de Educación a Distancia, Ciudad Universitaria s/n, 28040 Madrid. Correo-e: rmvalien@psi.uned.es. den, si son intensos, interferir en las actividades y desarrollo del niño y/o adolescente, aunque en menor grado que las fobias (Ollendick y King, 1994). Por otra parte, los miedos intensos que ocurren durante la infancia y/o adolescencia pueden derivar en fobias y otros problemas de ansiedad durante la edad adulta (Sandín, 1997, 1999; Valiente, Sandín y Chorot, 2002a).

La prevalencia de los miedos se ha estudiado habitualmente calculando el número de miedos que experimenta una 
población determinada de niños y/o adolescentes. En este sentido, se han indicado datos que sugieren la existencia de niveles elevados de prevalencia. Así por ejemplo, mientras que Ollendick, King y Frary (1989) y Gullone y King (1993) obtuvieron una prevalencia de 14 y 16,22 miedos, respectivamente, la prevalencia referida por Sandín, Chorot, Valiente y Santed (1998) fue ligeramente superior (19,8 miedos), y la obtenida por Shore y Rapport (1998) fue bastante mayor $(22,48$ miedos). En cada uno de estos estudios se emplearon distintas versiones revisadas del FSSC (Fear Survey Schedule for Children), ya que se utilizaron los cuestionarios FSSC-R (Ollendick et al., 1989; Sandín et al., 1998), FSSC-II (Gullone y King, 1993) y FSSC-HI (Shore y Rapport, 1998).

La mayoría de los estudios que han examinado mediante autoinforme la prevalencia de los miedos infantojuveniles en función del sexo han indicado que esta es mayor para los sujetos del sexo femenino que para los participantes del sexo masculino (Ollendick, Matson y Helsel, 1985; Ollendick et al., 1989; Gullone y King, 1993; Sandín et al., 1998). Lo cual es coherente con la mayoría de la evidencia obtenida con otros tipos de metodología diferentes a los procedimientos basados en cuestionarios de autoinforme (Ollendick, Hagopian y King, 1997; Gullone, 2000). También es coherente con los datos sobre diferencias sexuales en el autoinforme de miedos y fobias en personas adultas, los cuales suelen indicar siempre que las mujeres tienden a experimentar más miedos (tanto normativos como clínicos) que los hombres (Sandín y Chorot, 1995; Fredrikson, Annas, Fischer y Wik, 1996; Chapman, 1997; Sandín, 1999).

La prevalencia o frecuencia de miedos también se ha investigado analizando cuáles son los miedos más comunes (o prevalentes) en la población de niños y adolescentes. Esto se ha llevado a cabo a partir de la información obtenida en los cuestionarios de autoinforme examinando los elementos que son señalados por mayor número de sujetos como productores de alto nivel de miedo (i.e., elementos contestados con la máxima puntuación de miedo). De esta forma, pueden aislarse los miedos que son más comunes, para una población infantojuvenil determinada, entre todos los miedos (elementos) incluidos en el cuestionario concreto. Los estudios más relevantes en este sentido han utilizado el cuestionario FSSC-R; recientemente se han aportado también algunos datos basados en el empleo del FSSC-II. Ha sido habitual en este tipo de estudios resaltar los diez miedos más comunes.

Del análisis de las investigaciones que han abordado el estudio sobre cuáles son los miedos más prevalentes, se deriva que los miedos más comunes entre la población de niños y adolescentes son los relacionados con la dimensión de peligro y muerte. Esta conclusión general parece derivarse tanto de los estudios basados en el FSSC-R (Ollendick et al., 1989; McCathie y Spence, 1991; Ollendick, Yule y Ollier, 1991; Sandín et al., 1998; Shore y Rapport, 1998), como en el FSSC-II (Gullone y King, 1993; Burnham y Gullone, 1997).

Los diez miedos más comunes son prácticamente idénticos en las investigaciones realizadas con población europea (Ollendick et al., 1991; Sandín et al., 1998), norteamericana (Ollendick et al., 1989; McCathie y Spence, 1991), y australiana (Ollendick et al., 1989). Podría afirmarse que, con ligeras excepciones, todos estos miedos corresponden a la categoría o dimensión de miedos al peligro y a la muerte [las restantes dimensiones que pueden evaluarse a partir del FSSC-R son las relacionadas con miedos a lo desconocido, a los animales, al fracaso y a la crítica (dimensión social-eva- 
luativa), y a las situaciones de tipo médico]. Nueve de los diez miedos más comunes se repiten en estos cuatro estudios; i.e., miedos relacionados con ser atropellado por un coche o camión, no poder respirar, ser invadido por otro país, el fuego, caerse de lugares altos, que un ladrón entre en casa, los terremotos, la muerte o la gente muerta, y padecer una enfermedad grave. El restante miedo más común también coincide en los estudios con población británica (Ollendick et al., 1991) y en los de población española (Sandín et al., 1998), miedo que se refiere a la dimensión social-evaluativa (i.e., ser enviado al director del colegio).

Los miedos correspondientes a la dimensión social-evaluativa parecen tener mayor prevalencia en la población china (Dong, Xia, Lin, Yang, y Ollendick, 1995). En este estudio con población china se evidencia un mayor predominio que en los anteriores estudios de los miedos de tipo social, ya que aparecen entre los diez más comunes los temores relativos a obtener malas notas, ser castigado por el padre, suspender un examen, y presenciar las disputas paternas. Aunque cabría especular que tal diferencia podría deberse a factores de tipo étnico y/o cultural, resulta interesante subrayar que, incluso en el reciente estudio de Shore y Rapport (1998) con diferentes grupos étnicos (caucasianos, americanos de origen asiático, filipinos y hawaianos), se confirmó que los diez miedos más comunes, para todas y cada una de las cuatro submuestras, pertenecían a la categoría de miedos al peligro y a la muerte.

Los diez miedos más comunes también pueden estudiarse teniendo en cuenta las posibles diferencias asociadas al sexo. Curiosamente Ollendick et al. (1989) observaron una gran semejanza entre los diez miedos más comunes que exhibían los chicos y las chicas (coincidían en 9 de ellos). La diferencia radicaba en que el miedo a las serpientes, que aparecía en el grupo femenino, era sustituido en el grupo masculino por el miedo a obtener malas notas. Aparte, los autores indicaron que las niñas parecían preocuparse más por el miedo a perderse en un lugar extraño, y los niños por el miedo a una enfermedad grave. Este último resultado está de acuerdo con los hallazgos de McCathie y Spence (1991) que refirieron, entre los diez temores más comunes, el miedo a los microbios o a padecer una enfermedad grave como único del grupo de chicos (el miedo a la muerte o morirse resultó único del grupo de chicas).

En realidad, cuando se ha contrastado el contenido de los diez miedos más comunes en función del sexo, los resultados tienden a indicar que existen muy pocas diferencias (Ollendick, 1983; Ollendick et al., 1991; Gullone y King, 1993; Shore y Rapport, 1998). Los miedos más comunes suelen coincidir en los grupos de chicos y chicas con temores relacionados con el peligro y la muerte. Básicamente, lo que se observan son diferencias menores relacionadas con cambios en el orden, entre los diez más frecuentes; cambios que a veces afectan a que el miedo pertenezca o no a los diez más comunes, pero sin que por ello deje de ser bastante común. Por ejemplo, en el trabajo de Sandín et al. (1998), el temor a recibir una descarga eléctrica estaba en el grupo de chicos en tercer lugar, pero en el puesto undécimo en el grupo de chicas, lo cual implicaba que este miedo no apareciese entre los diez más comunes en la submuesta femenina, a pesar de ser el miedo inmediatamente posterior. Algo semejante ocurría con el miedo a que un ladrón entre en la casa: en el grupo de chicas estaba en cuarto lugar (por tanto entre los diez más frecuentes) y en el grupo de chicos en el duodécimo puesto (por tanto, fuera de los diez más frecuentes). 
En los estudios sobre los temores infantojuveniles, la intensidad del miedo es una variable que habitualmente se ha evaluado de forma paralela o complementaria a la prevalencia. Generalmente se ha empleado, bien el nivel global de miedo (i.e., intensidad obtenida a partir de la suma de los valores de todos los elementos del cuestionario), o bien el nivel de miedo en cada una de las cinco dimensiones del FSSC-R. Prácticamente todos los estudios relevantes basados en pruebas de autoinforme han encontrado que las niñas y las adolescentes exhiben niveles de miedo significativamente más elevados que los niños y los adolescentes, tanto en lo que concierne a los niveles globales de miedo como a los niveles de miedo en las diferentes dimensiones del FSSC-R (p.ej., Ollendick, 1983; Ollendick et al., 1985, 1989; McCathie y Spence, 1991; Gullone y King, 1993; King, Gullone y Ollendick, 1992; King, Hamilton y Ollendick, 1994; Dong et al., 1995; Burnham y Gullone, 1997; Sandín et al., 1998;). Algunos estudios que han empleado procedimientos de evaluación alternativos al FSSC-R también han documentado la existencia de niveles superiores de intensidad del miedo asociados a las niñas y las adolescentes (p.ej., Pelechano, 1981; Báguena y Chisbert, 1998).

Es decir, según estos estudios, en general las chicas siempre muestran niveles superiores de miedo, para cualquiera de las categorías de miedos de que se trate. Entre las pocas excepciones a esta regla caben mencionarse los resultados referidos por Ollendick et al. (1991) y Shore y Rapport (1998). Ollendick et al. (1991), partiendo de una muestra de niños británicos, no encontraron diferencias estadísticamente significativas entre ambos sexos para las dimensiones de miedos sociales (miedo al fracaso y a la crítica) y médicos. Shore y Rapport (1998), en una muestra de varias etnias, tampoco halla- ron diferencias significativas entre chicos $\mathrm{y}$ chicas para las dimensiones de miedos sociales anticipatorios y miedos de conformismo social. Aunque los resultados de estos dos estudios parecen indicar la inexistencia de diferencias de sexo en respuestas de miedo social, es mayor la evidencia que sugiere lo contrario, esto es, que existen diferencias sexuales, exhibiendo sistemáticamente las chicas mayores niveles de miedo social que los chicos. Por otra parte, esta conclusión general estaría de acuerdo con la literatura científica, aun cuando tales diferencias no parecen tan claras cuando se trata de miedos sociales clínicos en lugar de miedos sociales normativos (Sandín, 1997).

La finalidad del presente estudio consiste en examinar la prevalencia e intensidad de los miedos infantojuveniles teniendo en cuenta las posibles diferencias asociadas al sexo. Se pretende, así mismo, utilizar en el estudio una muestra amplia de participantes aplicando la versión española del cuestionario de autoinforme FSSC-R. Aunque este cuestionario es el más utilizado y validado internacionalmente, que nosotros sepamos sólo existen datos preliminares sobre la intensidad y prevalencia de los miedos obtenidos con la versión española del FSSC-R en una muestra limitada de sujetos (Sandín et al., 1998). Partiendo de la evidencia de la literatura, pronosticamos que: (1) el nivel de prevalencia debe ser elevado, pudiendo oscilar entre 10 y 20 miedos relevantes; (2) la prevalencia debería ser superior en las chicas que en los chicos; (3) los 10 miedos más comunes deberán pertenecer principalmente a la dimensión de miedos al peligro y a la muerte, lo cual debería ocurrir con independencia del sexo; y (4) el nivel de intensidad del miedo, tanto globalmente como según las diferentes dimensiones, debería asociarse positivamente al sexo femenino. 


\section{MÉTODO}

\section{Participantes}

La muestra fue de 1080 participantes normales (no clínicos ni con retraso mental), la cual estaba formada por 613 chicas y 467 chicos, con edades que oscilaban entre los 8 y los 18 años $(M=13,62, D T=$ 2,50 años). Las edades medias de los grupos femenino y masculino fueron, respectivamente, $13,76(D T=2,46)$ y 13,44 $(D T=2,54)$. No existían diferencias significativas en edad entre ambos grupos $(t$ $=-1,01, p>0,05)$. Los participantes poseian mayoritariamente niveles socioeconómicos medio o medio-bajo, y asistían regularmente a colegios públicos de ensenanza primaria o secundaria ubicados en la Comunidad de Navarra.

\section{Prueba psicológica y procedimiento}

Los participantes fueron evaluados en su propio centro docente, al cual asistían con regularidad durante el curso académico. De forma colectiva y supervisada por dos psicólogas, se les aplicó, junto a otras pruebas no incluidas en el presente estudio, el Cuestionario de Miedos para Niños-Revisado [Fear Survey Schedule for Children-Revised] (FSSC-R; Ollendick, 1983). Aplicamos la versión española de Chorot y Sandín (Sandín, 1997). Estudios recientes con la versión española del FSSC-R han aportado datos psicométricos que garantizan su validez, una elevada consistencia interna y una estructura de cinco factores similar a la establecida para la versión inglesa del cuestionario (Sandín y Chorot, 1998; Sandín et al., 1998; Valiente, 2001; Valiente, Sandín y Chorot, 2002b).

La versión española del FSSC-R, al igual que la versión en lengua inglesa, consta de 80 items de tres niveles de intensidad [1(nada), 2(un poco) y 3(mu- cho)]. De modo similar a como se ha hecho en los estudios con la versión inglesa del cuestionario, pueden calcularse la prevalencia y la intensidad de los miedos. La prevalencia de los miedos fue examinada siguiendo el mismo procedimiento sugerido por Ollendick et al. (1989), y aplicado por otros autores en el estudio de los miedos normativos (p.ej., Gullone y King, 1993; Sandín et al., 1998). De acuerdo con este procedimiento, la prevalencia del miedo se calculó sumando el número total de miedos (i.e., elementos del cuestionario) que el sujeto estimó como productores de mucho miedo. Nótese que este concepto difiere del concepto clásico de prevalencia (i.e., porcentaje de casos en una población). La intensidad se obtiene sumando los valores asignados por el participante a los elementos del cuestionario. Tanto para la prevalencia como para la intensidad, pueden obtenerse puntuaciones globales y puntuaciones separadas para las cinco dimensiones o subescalas del FSSC-R. El cuestionario incluye las 5 subescalas siguientes: (1) Miedos al fracaso y la crítica, (2) Miedos a pequeños animales y daños menores, (3) Miedos a los peligros físicos y muerte, (4) Miedos a lo desconocido, y (5) Miedos médicos.

Aunque los niveles globales de prevalencia y de intensidad del miedo se calcularon partiendo de los $\mathbf{8 0}$ items del cuestionario, las puntuaciones (prevalencia e intensidad) para las 5 subescalas se obtuvieron a partir de la forma depurada del mismo, forma que incluye únicamente los 55 items que resultaron ser relevantes para la construcción de dichas dimensiones (Valiente, 2001).

\section{RESULTADOS}

\section{Prevalencia de los miedos}

En la Tabla 1 se indican las medias y desviaciones típicas de las puntuaciones en 
prevalencia correspondientes al nivel global (FSSC-R total) y a las cinco dimensiones o subescalas, según la muestra total y los grupos de sexo. Calculamos un MANOVA de 2 (sexo: chicas, chicos) $\times 3$ (edad: 8-10 años, 11-14 años, 15-18 años) para las 6 variables dependientes (para la puntuación total del cuestionario de miedos y para las cinco dimensiones). Los resultados indicaron la existencia de efectos significativos debidos al sexo $[F(6,1069)=30,8, p<0,001]$ y a la edad, pero no a la interacción sexo $x$ edad [dada la no-interacción entre sexo y edad, en este estudio únicamente nos vamos a centrar en los datos relativos a las diferencias en función del sexo; los datos relacionados con las diferencias asociadas a la edad serán objeto de otro estudio].

Basándonos en estos resultados, efectuamos una serie de 6 ANOVAs univariados de $2 \times 3$, siendo interpretados únicamente los efectos principales del factor sexo, ya que no fue significativa la interacción entre el sexo y la edad en ninguno de los ANOVAs. En las comparaciones entre los dos grupos (i.e., chicas vs. chicos), tras los ANOVAs univariados las probabilidades se ajustaron a la corrección de Bonferroni (Keppel, 1982).

Como puede observarse a partir de los datos que presentamos en la Tabla 1, siste- máticamente las chicas obtienen puntuaciones más elevadas que los chicos. Estas diferencias fueron estadísticamente significativas para cada una de las variables dependientes estudiadas (i.e., número total de miedos, y número de miedos en las diferentes subescalas). Si nos atenemos a los valores de las medias y del estadístico $F$, aunque ocurren al mismo nivel de significación estadística, las diferencias parecen ser menos marcadas para los miedos de los tipos social (miedos al fracaso y a la crítica) y médico. Por otra parte, en general se observan niveles de prevalencia elevados, ya que los resultados indican que la media viene a oscilar entre 12 (chicos) y 17 (chicas) miedos importantes.

\section{Los miedos más comunes}

Siguiendo el método empleado por Ollendick et al. (1989) para analizar los miedos más comunes, se calcularon los porcentajes de sujetos que estimaron cada ítem del cuestionario con el valor de mayor intensidad (i.e., con valor de 3). En la Tabla 2 indicamos los diez miedos más comunes (i.e., estimados por el mayor número de sujetos con la máxima intensidad) para la muestra total y para los grupos de sexo.

Tabla 1. Prevalencia de los miedos para la muestra total y según el sexo

\begin{tabular}{|c|c|c|c|c|c|c|c|}
\hline \multirow[b]{3}{*}{ Variable del FSSC-R } & & & \multicolumn{4}{|c|}{ Sexo } & \multirow[b]{3}{*}{$F(1,1079)$} \\
\hline & \multicolumn{2}{|c|}{$\begin{array}{c}\text { Total } \\
(N=1080)\end{array}$} & \multicolumn{2}{|c|}{$\begin{array}{c}\text { Femenino } \\
(n=613)\end{array}$} & \multicolumn{2}{|c|}{$\begin{array}{c}\text { Masculino } \\
(n=467)\end{array}$} & \\
\hline & Media & $D T$ & Media & $D T$ & Media & $D T$ & \\
\hline \multicolumn{8}{|l|}{ FSSC-R (subescalas): } \\
\hline Miedos a los animales (10) & 1,72 & 1,85 & 2,23 & 2,03 & 1,06 & 1,32 & $117,1^{*}$ \\
\hline Miedos al fracaso y a la crítica (16) & 2,79 & 2,90 & 3,02 & 3,00 & 2,46 & 2,74 & $20,9^{\star}$ \\
\hline Miedos al peligro y a la muerte (12) & 6,43 & 3,13 & 7,24 & 2,98 & 5,37 & 3,00 & $108,3^{\star}$ \\
\hline Miedos a lo desconocido (12) & 1,32 & 1,83 & 1,67 & 1,94 & 0,86 & 1,65 & $85,8^{\star}$ \\
\hline Miedos médicos (5) & 0,76 & 1,09 & 0,92 & 1,17 & 0,55 & 1,17 & $33,4^{*}$ \\
\hline FSSC-R total (80) & 14,67 & 9,46 & 16,94 & 9,51 & 11,67 & 8,52 & $120,1^{*}$ \\
\hline
\end{tabular}

Nota: Entre paréntesis se indica el número de elementos de cada dimensión o subescala.

${ }^{\star} p<0,001$ (se aplicó la corrección de Bonferroni). 
Tabla 2. Los 10 miedos más comunes para la muestra total y según el sexo (\% de sujetos que estimaron con la máxima intensidad cada miedo que se indica)

\begin{tabular}{lccc}
\hline & & \multicolumn{2}{c}{ Sexo } \\
\cline { 4 - 4 } ftem del FSSC-R & $\begin{array}{c}\text { Total } \\
(N=1080)\end{array}$ & $\begin{array}{c}\text { Femenino } \\
(n=613)\end{array}$ & $\begin{array}{c}\text { Masculino } \\
(n=\mathbf{4 6 7})\end{array}$ \\
\hline 20. Bombardeos & 75,8 & $\mathbf{8 3 , 6}$ & 65,3 \\
76. No poder respirar & 74,1 & $\mathbf{7 8 , 6}$ & $\mathbf{6 7 , 8}$ \\
41. Ser atropellado & 65,5 & $\mathbf{6 7 , 8}$ & 62,5 \\
26. Ladrones & 65,4 & $\mathbf{7 5 , 7}$ & $\mathbf{5 1 , 3}$ \\
70. Enfermedad grave & 53,9 & 59,9 & 45,6 \\
59. Descarga eléctrica & 53,3 & $\mathbf{5 7 , 5}$ & $\mathbf{4 7 , 5}$ \\
15. Ser enviado al director & 48,2 & $\mathbf{4 9 , 5}$ & $\mathbf{4 6 , 4}$ \\
58. Caerse de lugares altos & 47,8 & 51,6 & $\mathbf{4 2 , 6}$ \\
9. La muerte/gente muerta & 43,3 & $\mathbf{5 1 , 6}$ & $\mathbf{3 1 , 9}$ \\
72. Los terremotos & 43,1 & $\mathbf{4 7 , 7}$ & $\mathbf{3 6 , 7}$ \\
\hline
\end{tabular}

Nota: El asterisco $\left({ }^{*}\right)$ indica que el miedo no se encontraba entre los diez más comunes en ese grupo concreto.

Del análisis de la Tabla 2 se desprende que la casi totalidad (9 de 10) de los temores más comunes pertenecen a la dimensión de miedos al peligro y a la muerte. Uno de los diez miedos más comunes pertenece al factor de miedos al fracaso y a la crítica (ítem 15). Un aspecto importante a resaltar es la gran concordancia que existe entre los 10 miedos más comunes a través de los grupos de sexo. Así, los chicos y las chicas coincidieron en 9 de los miedos más comunes (los 10 miedos del grupo de chicas coincide con los 10 de la muestra total). En el grupo de chicos, el ítem 9 (miedo a la muerte o a la gente muerta) no se encontraba entre los diez miedos más señalados. Sí se encontraba, en cambio, el ítem 29 (malas notas), elegido por el $41,6 \%$ de los chicos.

\section{Intensidad de los miedos}

La intensidad de los miedos se calculó sumando los valores estimados por los sujetos en los elementos del cuestionario. De este modo, se obtuvo una puntuación total de intensidad de miedo y puntuaciones en las diferentes dimensiones (subescalas). Los análisis estadísticos efectuados para examinar las diferencias asociadas al sexo fueron similares a los llevados a cabo para estudiar las variables de prevalencia de los miedos (véase atrás).

El MANOVA indicó la existencia de efectos significativos asociados al sexo $[F(6,1037)=41,6, p<0,001]$ y a la edad. Como ocurría para el caso de la prevalencia, no encontramos efectos significativos asociados a la interacción entre el sexo y la edad. Las medias, desviaciones típicas y resultados de los sucesivos ANOVAs relacionados con las diferencias entre los grupos de sexo están indicados en la Tabla 3. Como se expone en dicha tabla, se obtuvieron sistemáticamente diferencias significativas entre los grupos de chicos y chicas, tanto para la intensidad total de miedo como para la intensidad en cada una de las cinco dimensiones. No obstante, de modo similar a lo que ocurría para la prevalencia, si nos atenemos a los valores de las medias y del estadístico $F$, aunque ocurren al mismo nivel de significación estadística, las diferencias parecen ser menos marcadas para los miedos de los tipos social (miedos al fracaso y a la crítica) y para los miedos médicos. 
Tabla 3. Intensidad de los miedos según el sexo

\begin{tabular}{|c|c|c|c|c|c|c|c|}
\hline \multirow[b]{3}{*}{ Variable del FSSC-R } & & & \multicolumn{4}{|c|}{ Sexo } & \multirow[b]{3}{*}{$F(1,1048)$} \\
\hline & \multicolumn{2}{|c|}{$\begin{array}{c}\text { Total } \\
(N=1080)\end{array}$} & \multicolumn{2}{|c|}{$\begin{array}{c}\text { Femenino } \\
(n=613)\end{array}$} & \multicolumn{2}{|c|}{$\begin{array}{c}\text { Masculino } \\
(n=467)\end{array}$} & \\
\hline & Media & $D T$ & Media & $D T$ & Media & $D T$ & \\
\hline \multicolumn{8}{|l|}{ FSSC-R (subescalas): } \\
\hline Miedos a los animales $(10-30)$ & 16,6 & 4,00 & 18,1 & 3,97 & 14,8 & 3,20 & $195,2^{\star}$ \\
\hline Miedos al fracaso y a la crítica $(16-48)$ & 28,8 & 5,84 & 29,5 & 5,75 & 28,1 & 5,86 & $20,7^{\star}$ \\
\hline Miedos al peligro y a la muerte (12-36) & 28,7 & 4,61 & 30,2 & 3,99 & 26,9 & 4,71 & $114,4^{*}$ \\
\hline Miedos a lo desconocido (12-36) & 18,2 & 4,38 & 19,3 & 4,36 & 16,7 & 3,97 & $124,5^{*}$ \\
\hline Miedos médicos (5-15) & $\mathbf{8 , 3}$ & 2,36 & 8,7 & 2,41 & 7,6 & 2,17 & $51,4^{*}$ \\
\hline FSSC-R total (80) & 133,5 & 20,52 & 139,5 & 19,44 & 125,8 & 19,31 & $140,1^{\star}$ \\
\hline
\end{tabular}

Nota: Entre paréntesis se indica el rango de posibles valores para cada variable dependiente.

${ }^{*} p<0,001$ (se aplicó la corrección de Bonferroni).

\section{DISCUSIÓN}

A partir del presente estudio hemos intentado examinar en una muestra amplia de niños y adolescentes las diferencias sexuales en la prevalencia e intensidad de los miedos normativos. De acuerdo con los datos de la literatura internacional sobre los miedos infantojuveniles habíamos hipotetizado la existencia de: (1) niveles elevados de prevalencia; (2) niveles de prevalencia e intensidad superiores en las chicas que en los chicos, tanto a nivel general como en función de las diferentes dimensiones de miedos; y (3) mayor prevalencia, tanto en los chicos como en las chicas, de los miedos pertenecientes a la dimensión de miedos a los peligros físicos y a la muerte. En términos generales podríamos afirmar que los datos proporcionan apoyo empírico consistente con estas hipótesis.

Los niveles de prevalencia obtenidos indican que los niños y los adolescentes refieren tener un número alto de miedos relevantes, oscilando entre 12 miedos en los chicos y 17 miedos en las chicas. Estos datos confirman los resultados obtenidos con niños y adolescentes de otras culturas como la norteamericana (Ollendick et al., 1989), la australiana (Ollendick et al., 1989; Gullone y King, 1993), la británica
(Ollendick et al., 1991), la china (Dong et al., 1994) y otros grupos étnicos diversos (Shore y Rapport, 1998). Así mismo, los resultados son consistentes con nuestros datos iniciales obtenidos a partir de una muestra de niños españoles (Sandín et al., 1998). Por tanto, podríamos decir que, también en el marco de la cultura española, los miedos subclínicos son bastante frecuentes en la población infantil.

Nuestros resultados sobre la prevalencia de los miedos proporcionan apoyo a nuestra hipótesis de que las chicas obtendrían puntuaciones superiores a los chicos, tanto en la puntuación total de miedos como en el número de miedos correspondientes a todas y cada una de las cinco dimensiones de los miedos. Las diferencias en los niveles de prevalencia entre las chicas y los chicos siempre son estadísticamente significativas para todas las variables analizadas. Tales resultados son congruentes con la literatura científica sobre las diferencias de género en miedos y fobias (Echeburúa, 1993; Fredrikson et al., 1996; Chapman, 1997; Sandín, 1997, 1999; Botella y Gallardo, 1999; Essau, Conradt, y Petermann, 1999), corroboran los datos preliminares de Sandín et al. (1998) para niños de 9-11 años, y apoyan la evidencia obtenida con las versiones inglesas del FSSC-R (Ollendick et al., 1985, 1989; 
McCathie y Spence, 1991; Dong, Yang y Ollendick, 1994; Shore y Rapport, 1998) y FSSC-II (Gullone y King, 1993; Gullone, 2000).

En relación con la intensidad de los miedos, los datos obtenidos en el presente estudio indican que, al igual que ocurría para la prevalencia, las chicas informan mayores niveles de miedo que los chicos. Las diferencias son significativas tanto para la intensidad total de miedo, como para la intensidad de miedo en cada una de las diferentes dimensiones. Estos resultados son consistentes con el grueso de la literatura científica sobre los miedos, la cual ha sugerido de forma bastante sistemática que, con independencia de la edad, las chicas informan poseer mayores niveles de miedo que los chicos (Bamber, 1974; Ollendick et al., 1985, 1989, 1991; Gullone y King, 1993; Dong et al., 1994; Burnham y Gullone, 1997; Sandín et al., 1998; Scherer y Nakamura, 1968). Aunque en limitados estudios no se han encontrado diferencias entre chicos y chicas para los miedos sociales (Ollendick et al., 1991; Shore y Rapport, 1998) y médicos (Ollendick et al., 1991), nuestros datos sugieren que las diferencias son extensibles a las cinco categorías de miedos estudiadas.

Finalmente, en relación con los miedos más comunes, habíamos hipotetizado que estos deberían pertenecer la dimensión de miedos al peligro y a la muerte. Tal y como se indica en la Tabla 2, la mayoría de los 10 miedos más comunies ( 9 para ser exactos) pertenecen a dicha dimensión. Vemos que este fenómeno se produce con independencia del sexo, lo cual es consistente con los datos publicados en la literatura sobre esta cuestión, ya que en general se ha venido señalando que, con la posible excepción del estudio con población china de Dong et al. (1995; predominaban también los miedos de tipo social-evaluativo), los miedos más comunes son los relacionados con el peligro y la muerte (Ollendick et al., 1989, 1991; McCathie y Spence, 1991; Gullone y King, 1993; Burnham y Gullone, 1997; Sandín et al., 1998). De acuerdo con la teoría de preparación de las fobias (Öhman, 1986; Öhman y Mineka, 2001), este es el resultado que cabría esperar dada la especial relevancia que estos miedos poseen para la supervivencia.

En suma, a partir del presente estudio hemos constatado que la prevalencia de los miedos subclínicos es elevada en una muestra amplia de la población de niños y adolescentes españoles. Las diferencias entre chicos y chicas en prevalencia e intensidad de los miedos están de acuerdo con el grueso de la literatura sobre las diferencias sexuales en el autoinforme y manifestación de los miedos y las fobias. La naturaleza de los 10 miedos más comunes, similar en ambos sexos, sugiere que los miedos relacionados con los peligros mayores y con la muerte podrían desempeñar un papel adaptativo preponderante durante las etapas de la infancia y la adolescencia. Como nota final, desearíamos indicar que el presente trabajo proporciona evidencia transcultural sólida sobre la prevalencia e intensidad de los miedos infantojuveniles, basada en la versión española del FSSC-R, y congruente con la evidencia internacional obtenida a partir de este mismo cuestionario o versiones derivadas. Como hemos documentado en nuestro anterior trabajo (Valiente et al., 2002b), tanto la prevalencia como la intensidad de los miedos pueden estar asociadas a ciertas variables de vulnerabilidad, tales como la sensibilidad a la ansiedad, el rasgo de ansiedad y el afecto negativo. Otro aspecto importante que merece ser estudiado, y que posiblemente esté relacionado con la prevalencia e intensidad de los miedos infantojuveniles, viene dado por las posibles diferencias asociadas a los grupos de edad. 


\section{REFERENCIAS BIBLIOGRÁFICAS}

Báguena, M.J., y Chisbert, M.J (1998). El género como modulador de la evolución psicológica de los miedos. Análisis y Modificación de Conducta, 24, 329-451.

Bamber, J.H. (1974). The fears of adolescents. The Journal of Genetic Psychology, 125, 127-140.

Botella, C., y Gallardo, M. (1999). La fobia social. Madrid: Klinik.

Burnham, J.J., y Gullone E. (1997). The Fear Survey Schedule for Children-II: A psychometric investigation with American data. Behaviour Research and Therapy, 35, 165-173.

Chapman, T.F. (1997). Epidemiology. En G.C.L. Davey (Ed.), Phobias: A handbook of theory, research and treatment (pp. 415434). Chichester: Wiley.

Dong, Q., Xia, Y., Lin, L., Yang, B., y Ollendick, T.H. (1995). The stability and prediction of Fears in Chinese Children and Adolescents: a one-year follow-up. Journal of Child Psychology and Psychiatry, 36, 819-831.

Dong, Q., Yang, B., y Ollendick, T.H. (1994). Fears in Chinese Children and Adolescents and their relations to Anxiety and Depression. Journal of Child Psychology and Psychiatry, 35, 351-363.

Echeburúa, E. (1993) Trastornos de ansiedad en la infancia. Madrid: Pirámide.

Essau, C.A., Conradt, J., y Petermann, F. (1999). Frequency and comorbidity of social phobia and social fears in adolescents. Behaviour Research and Therapy, 37, 831-843.

Fredrikson, M., Annas, P., Fischer, H., y Wik, G. (1996). Gender and age differences in the prevalence of specific fears and phobias. Behaviour Research and Therapy, 34, 33-39.

Gullone, E. (2000). The development of normal fear: A century of research. Clinical Psychology Review, 20, 429-451.

Gullone, E., y King, N.J. (1993). The Fears of Youth in the 1990s: Contemporary Normative Data. The Journal of Genetic Psychology, 154, 137-153.

Keppel, G. (1982). Design and analysis: $A$ researcher's handbook (2nd ed.). Englewood Cliffs, NJ: Prentice-Hall.
King, N.J., Gullone, E., y Ollendick, T.H. (1992). Manifest Anxiety and Fearfulness in Children and Adolescents. The Journal of Genetic Psychology, 153, 63-73.

King, N.J., Hamilton, D.I., y Ollendick, T.H. (1994). Children's phobias: a behavioral perspective. Nueva York: Wiley.

McCathie, H., y Spence, S.H. (1991). What is the Revised Fear Survey Schedule for Children measuring? Behaviour Research and Therapy, 29, 495-502.

Öhman, A. (1986). Face of beast and fear the face: Animal and social phobias as prototypes for evolutionary analyses of emotion. Psychophysiology, 23, 123-145.

Öhman, A., y Mineka, S. (2001). Fears, phobias, and preparedness: Toward an evolved module of fear and fear learning. Psychological Review, 108, 483-522.

Ollendick, T.H. (1983). Reliability and validity of the Revised Fear Survey Schedule for Children (FSSC-R). Behaviour Research and Therapy, 21, 685-692.

Ollendick, T.H., y King, N. (1994). Fears and their level of interference in adolescents. Behavior Research and Therapy, 32, 635638.

Ollendick, T.H., Hagopian, L.P., y King, N.J. (1997). Specific phobias in children. En G.C.L. Davey (Ed.), Phobias: $A$ handbook of theory, research and treatment (pp. 202224). Chichester: Wiley.

Ollendick, T.H., King, N.J., y Frary, R.B. (1989). Fears in children and adolescents: Reliability and generalizability across gender, age and nationality. Behaviour Research and Therapy, 27, 19-26.

Ollendick, T.H., Matson, J.L., y Helsel, W.J. (1985). Fears in children and adolescents: Normative data. Behaviour Research and Therapy, 23, 465-467.

Ollendick, T.H., Yule, W., y Ollier, K. (1991). Fears in British Children and their relationship to Manifest Anxiety and Depression. Journal of Child Psychology and Psychiatry, 32, 321-331.

Pelechano, V. (1981). Miedos infantiles y.terapia familiar-natural. Valencia: Alfaplus.

Sandín, B. (1997). Ansiedad, miedos y fobias en niños y adolescentes. Madrid: Dykinson.

Sandín, B. (1999). Las fobias especificas. Madrid: Klinik. 
Sandín, B., y Chorot, P. (1995). Síndromes clínicos de la ansiedad. En A. Belloch, B. Sandín y F. Ramos (Eds.), Manual de psicopatología (Vol. 2, pp. 81-112). Madrid: McGraw-Hill.

Sandín, B., y Chorot, P. (1998). Dimensiones de los miedos en niños: Estructura factorial del FSSC-R. Psiquis, 19, 23-32.

Sandín, B., Chorot, P., Valiente, R.M., y Santed, M.A. (1998). Frecuencia e intensidad de los miedos en los niños: Datos normativos. Revista de Psicopatología y Psicología Clinica, 3, 15-25.

Scherer, M.W., y Nakamura, C.Y. (1968). A fear survey schedule for children. Behaviour Research and Therapy, 6, 172-182.

Shore, G.N., y Rapport, M.D. (1998). The Fear
Survey Schedule for Children-Revised (FSSC-HI): Etnocultural variations in children's fearfulness. Journal of Anxiety Disorders, 12, 437-461.

Valiente, R.M. (2001). Estructura y adquisición de los miedos en la infancia y la adolescencia. UNED, Madrid (Tesis Doctoral, no publicada).

Valiente, R.M., Sandín, B., y Chorot, P. (2002a). Miedos en la infancia y la adolescencia. Madrid: UNED.

Valiente, R.M., Sandín, B., y Chorot, P. (2002b). Miedos comunes en niños y adolescentes: Relación con la sensibilidad a la ansiedad, el rasgo de ansiedad, la afectividad negativa y la depresión. Revista de Psicopatología y Psicología Clínica, 7, 61-70. 